\title{
ENDOVASCULAR TREATMENT OF SUBARACHNOID HAEMORRHAGE: FIRST TIME AT DHAKA MEDICAL COLLEGE HOSPITAL
}

KHAN SU ${ }^{1}$ SIDDIQUI MMR ${ }^{2}$, RAHMAN KM ${ }^{1}$, HOQUE MA ${ }^{3}$, MONDOL MBA ${ }^{3}$, DAISY S ${ }^{3}$, HABIB M $^{4}$ HUSAIN S ${ }^{5}$, MOHAMMAD QD ${ }^{4}$

\begin{abstract}
:
Subarachnoid hemorrhage (SAH) due to rupture aneurysm is a neurologic catastrophe with a very high risk of rebleeding if the aneurysm is not treated. Endovascular coiling has becoming common and has replaced neurosurgical clipping as the treatment of choice. A 48-year-old man came to us with the history of SAH from ruptured cerebral arterial aneurysm and for the first time in the history of Dhaka Medical College Hospital, we had successfully treated by Endovascular coiling.
\end{abstract}

Key words: Subarachnoid haemorrhage (SAH), cerebral DSA, surgical clipping, endovascular coiling.

(J Dhaka Med Coll. 2008; 17(2) : 131-133)

\section{Introduction:}

Subarachnoid haemorrhage (SAH) accounts for approximately $1-7 \%$ of all strokes ${ }^{1}$ that has an incidence of approximately 6 to 15 per 100,000 people per year. ${ }^{2}$ Cerebral aneurysms rupture is the most frequent $(75-85 \%)$ cause of SAH. ${ }^{3}$ It is a medical emergency and can lead to death or severe disability-even when recognized and treated at an early stage. Up to half of all cases of SAH are fatal and 10-15\% die before reaching a hospital and those who survive often have neurological or cognitive impairment. ${ }^{3}$ Diagnosing of the SAH can be challenging, and treatment is complex, sophisticated, multidisciplinary. Patients with aneurysmal SAH are at very high risk of rebleeding if the aneurysm is not treated. The standard treatment for several decades has been surgical clipping of the neck of the aneurysm. In recent years, an alternative, endovascular treatment to occlude the aneurysm, has become more common.

\section{Case report:}

A 48-yrar-old, normotensive, non diabetic, right handed, man admitted in the department of Neurology, Dhaka Medical College Hospital with the history of unconsciousness following sudden severe headache along with vomiting and blurring of vision about 7 months back. During that period he was admitted in a hospital and diagnosed as a case of SAH. He recovered with medical treatment without any residual neurological deficit. Clinical examinations revealed nothing abnormalities. His all routine investigations were within normal limit, but previous Computerized Tomography (CT) scan of head showed features of subarachnoid haemorrhage (Fig.1). For further evaluation, we had done Cerebral

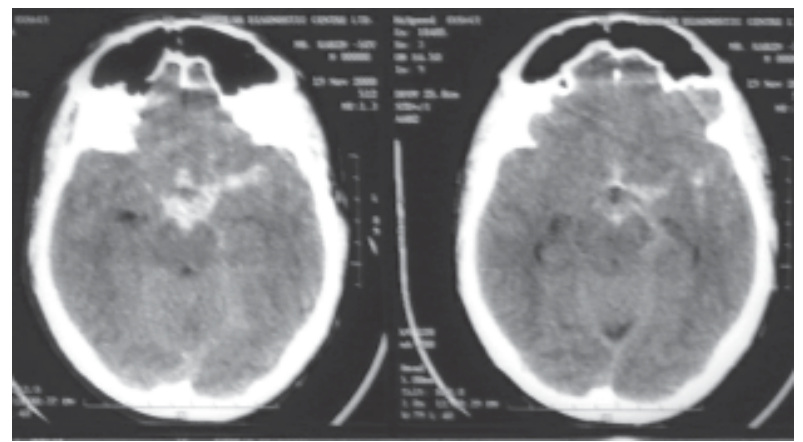

Fig.-1: Non contrast CT scan of the brain showing, hyper intense shadows in the basal cisterns \& sylvian fissures due to subarachnoid haemorrhage.

1. Assistant Professor, Department of Neurology, Dhaka Medical College Hospital, Dhaka, Bangladesh.

2. Postgraduate resident, Department of Medicine, Dhaka Medical College Hospital, Dhaka, Bangladesh.

3. Associate Professor, Department of Neurology, Dhaka Medical College Hospital, Dhaka, Bangladesh.

4. Professor, Department of Neurology, Dhaka Medical College Hospital, Dhaka, Bangladesh.

5. Professor \& Chief, Department of Neuroendovascular therapy, Sir Ganga Ram Hospital, New Deilhi, India.

Address of correspondence: Dr. Sharif Uddin Khan 
Digital Subtraction Angiogram (DSA) at our hospital and revealed a Bi-lobbed aneurysm at the junction of left internal carotid artery and left posterior communicating artery (Lt. P Com) (Fig.2). For the first time at Dhaka Medical College hospital, we attempted endovascular approach for the treatment of this aneurysm.

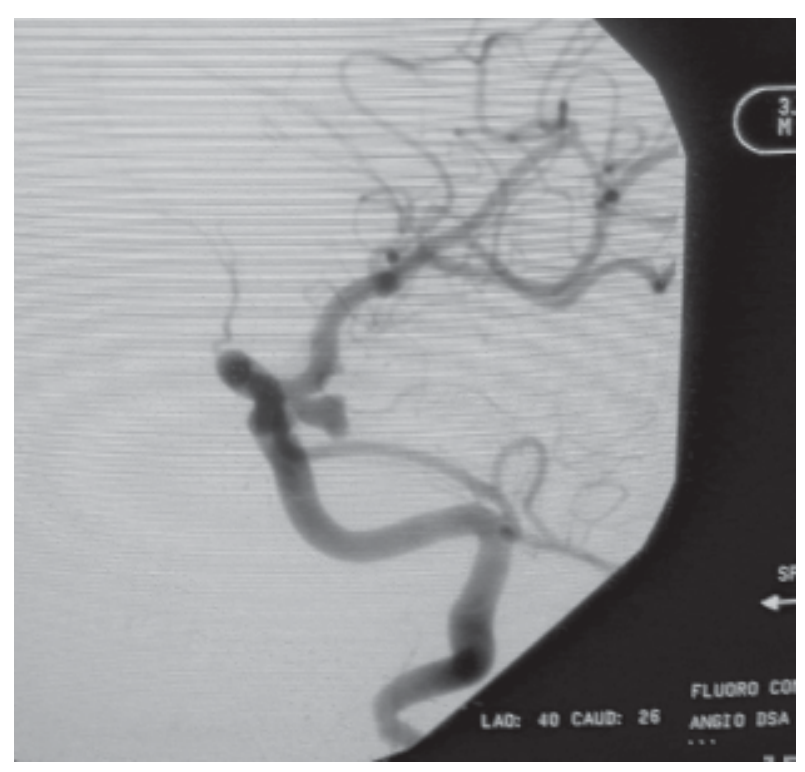

Fig.-2: CerebralDSA showing an aneurysm (black arrow) at the junction of left internal carotid artery and left posterior communicating artery.

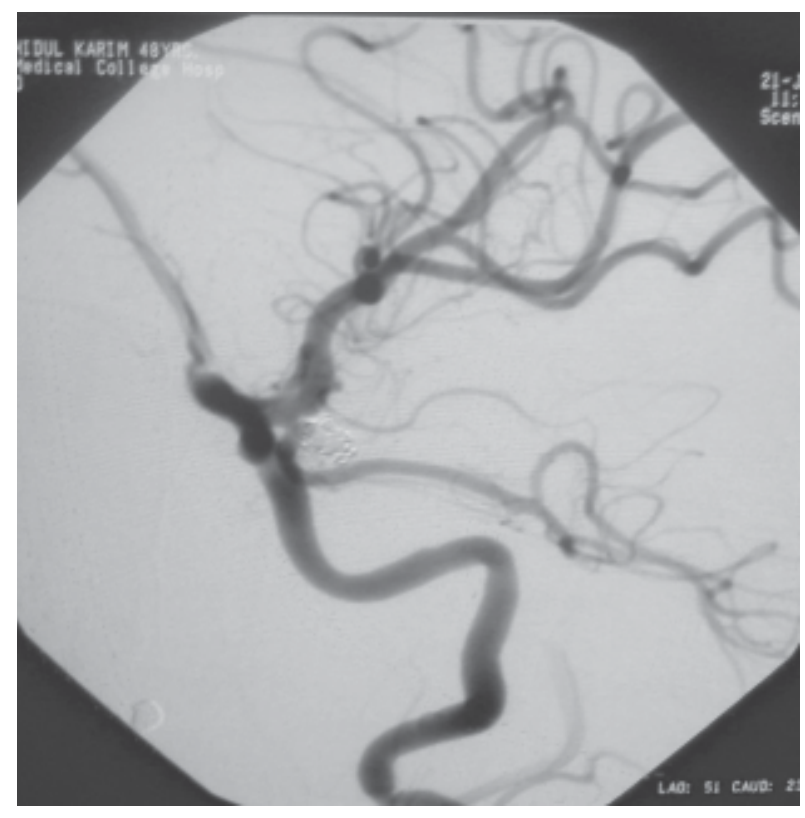

Fig.-3: Cerebral DSA showing, complete disappearance of the previous aneurism (black arrow) after endovascular treatment by coiling.
A $6 \mathrm{~F}$ guiding catheter was placed in left vertebral artery (LVA). Then Transend Excell 14 was placed upto the neck of the aneurysm along with Transend 10. Tip of the microcatheter was placed within the aneurysm sac and GDC 10 soft $(6 \times 11 \mathrm{~mm})$ coil was deployed and made a basket. Then second coil GDC10 soft $(4 \times 10 \mathrm{~mm})$ and GDC10 soft $(2 \times 6)$ were deployed and the aneurysm sac was totally occluded. Check angiogram reveals complete embolization of the aneurysm sac (Fig.3). Patient was stable and no peri-procedural complication was noted. In subsequent follow up visit he was completely symptoms free and living a healthy life.

\section{Discussion:}

Bleeding on the surface of the brain is called a subarachnoid haemorrhage. ${ }^{4}$ The bleeding usually comes from the rupture of a weak spot (aneurysm) in an artery carrying blood to the brain. ${ }^{4}$ It occurs in relatively young patients: half the patients are younger than 55 years of age and it carries a poor prognosis. ${ }^{5}$ Half the patients die within one month of the haemorrhage. ${ }^{4}$ SAH causes an unexpected, sudden, thunderclap headache and may lead to loss of consciousness. The patient reports the worst headache of his or her life. Hours after onset of symptoms, neurologic examination may reveal neck rigidity, cranial neuropathy (third or sixth cranial nerve most commonly), or other localized neurologic deficit (aphasia, hemiparesis); however, major neurologic signs generally are absent. Seizures may develop in less than $10 \%$ cases, particularly in those who had a subdural hematoma or cerebral infarction during their hospital course. ${ }^{6}$

Computed tomography is the first-line diagnostic procedure in patients with suspected SAH. Historically, CT has $90 \%$ to $95 \%$ sensitivity for recent SAH; with modern CT equipment, sensitivity is closer to $98 \%{ }^{7} \mathrm{CSF}$ study for the presence of xanthochromia, the yellow tinge in CSF caused by the breakdown products of hemoglobin, is the gold standard for diagnosis of $\mathrm{SAH}$, with a sensitivity greater than $99 \%$. Xanthochromia is present as early as 6 hours after SAH and along with bilirubin 
remains detectable until about 2 to 3 weeks after SAH. ${ }^{8}$ Once SAH is confirmed, 4-vessel cerebral angiography is needed to identify and characterize the source of hemorrhage. Noninvasive imaging techniques such as magnetic resonance angiography (MRA) and CT angiography used for making fundamental treatment decisions for intracranial aneurysm. ${ }^{9}$ Recently cerebral Digital subtraction angiogram (DSA) is the gold standard investigation. Definitive treatment of the aneurysm is recommended as soon as possible to minimize risk of recurrent aneurysmal hemorrhage. Currently, the 2 primary options for aneurysm treatment are craniotomy and aneurysm neck clipping or transvascular endosaccular coiling. ${ }^{9}$ In many institutes endovascular coiling has replaced neurosurgical clipping as the treatment of choice, if coiling is technically feasible. The major advantage of endovascular coiling is that a craniotomy is avoided and recovery after the procedure is more rapid. ${ }^{4}$ In many observational studies on endovascular treatment in patients with $\mathrm{SAH}$, the risk of rebleeding in the initial weeks after coiling is very low. ${ }^{4} \mathrm{SAH}$ showed a reduction of poor outcome rate after treatment by coiling in compared with surgical clipping. ${ }^{4} \mathrm{~A}$ disadvantage of coiling is that aneurysms are more often incompletely treated $(90 \%$ to $100 \%$ obliteration) and carry a rare risk for reopening. ${ }^{4}$ For patients in good clinical condition with ruptured aneurysms, if the aneurysm is considered suitable for both surgical clipping and endovascular treatment, coiling is associated with a better outcome.

\section{References:}

1. Feigin VL, Rinkel GJ, Lawes CM, et al. Risk factors for subarachnoid hemorrhage: an updated systematic review of epidemiological studies. Stroke. 2005; 36(12): 2773-80.

2. Linn FHH, Rinkel GJE, Algra A, van Gijn J. Incidence of subarachnoid hemorrhage: role of region, year and rate of computed tomography: a meta-analysis. Stroke 1996;27:625-9.

3. Van Gijn J, Kerr RS, Rinkel GJ et al. Subarachnoid haemorrhage. Lancet. 2007;369(9558):306-18.

4. Schaaf IVD, Algra A, Wermer M. Endovascular coiling versus neurosurgical clipping for patients with aneurysmal subarachnoid haemorrhage (Review). Cochrane Database of Systematic Reviews. 2005; 4: 1-29.

5. The ACROSS Group. Epidemiology of aneurysmal subarachnoid hemorrhage in Australia and New Zealand. Incidence and case fatality from the Australasian Cooperative Research on Subarachnoid Hemorrhage Study. Stroke. 2000; 31: 1843-50.

6. Claassen J, Peery S, Kreiter KT, et al. Predictors and clinical impact of epilepsy after subarachnoid hemorrhage. Neurology 2003; 60: 208-14.

7. Van Gijn J, Rinkel GJ. Subarachnoid haemorrhage: diagnosis, causes and management. Brain 2001; 124(2): 249-78.

8. Iversen SA. CSF spectrophotometry in the diagnosis of subarachnoid haemorrhage [letter]. J Clin. Pathol. 2002; 55: 640.

9. Wijdicks EFM, Kallmes DF, Manno EM et al. Subarachnoid Hemorrhage: Neurointensive Care and Aneurysm Repair. Mayo Clin Proc. 2005; 80(4): 550-9. 